Revista Educación 25(2): 101-111, 2001

\title{
REFLEXIONES EN TORNO A LA EDUCACIÓN RELIGIOSA: ELEMENTOS PARA UNA PROPUESTA DESDE LA PSICOLOGÍA DE LA RELIGIÓN
}

José Miguel Rodríguez García

Resumen: Este trabajo presenta, críticamente, los fundamentos de la educación religiosa en Costa Rica. A tal fin, retoma elementos conceptuales de la psicología de la religión y de la psicología del desarrollo bumano; en particular, de la psicología del desarrollo moral. Principalmente, reflexiona en torno al desarrollo de la fe y al juicio moral sobre la educación religiosa en Costa Rica. Finalmente, critica algunos elementos que plantean una educación religiosa tendiente a desarrollar la fe junto con el juicio moral, además de ofrecer una visión universalista sobre el fenómeno religioso.

\section{Introducción}

Para analizar la educación religiosa en Costa Rica, no se puede prescindir de que éste es un país esencialmente católico, apostólico y romano, como reza la Constitución, sin impedir el desarrollo de otros cultos, siempre y cuando no atenten contra la moral ni las buenas costumbres (Constitución Política de la República de Costa Rica, 1992).

Desde este punto de vista, se espera que, en las escuelas públicas de Costa Rica, la educación religiosa esté orientada hacia una perspectiva católica ${ }^{1}$.

Las clases de religión, como son conocidas por los estudiantes de escuelas y colegios, se basan, única y exclusivamente, sobre una revisión de la doctrina religiosa católica, para que comiencen a conocerla.

Lo anterior puede ser observado en los propósitos desde los cuales se parte, en Costa Rica, para tal tipo de educación religiosa:

1. Que los estudiantes aprendan el mensaje cristiano y adquieran bases religiosas y éticas.

2. Que se enriquezcan los procesos de enseñanza desde la perspectiva de la fe cristiana.

3. Que se faciliten contenidos teóricos y evangelizadores. 
4. Que se enseñe el reconocimiento de la dignidad de la persona y su aplicación en la vida como ciudadano.

5. Que se promueva la valoración de la familia, la persona humana, la situación social, la cultura, la interiorización de principios y valores cristianos.

6. Que se impulsen acciones que mejoren las condiciones de vida de las personas.

7. Que se promuevan, en los educandos, relaciones auténticas consigo mismos, con los demás y con Dios, desde la perspectiva del compromiso cristiano (Ministerio de Educación Pública, 1996).

De estos propósitos, se entresaca que la educación religiosa costarricense espera, de sí misma, promover, en los alumnos, el conocimiento de las bases de la doctrina de la Iglesia Católica con repercusiones positivas en la conducta individual dentro de lo social; conducta guiada por la ética y los valores cristianos.

Tal propuesta no dista mucho de la concepción de la Iglesia Católica, cuyos fundamentos doctrinales promueven y mueven la educación religiosa católica en las distintas partes del globo, sobre la base del cuestionamiento de qué deba ser y hacer la educación, aspecto central de la última versión del Catecismo de la Iglesia Católica (1992).

En su prólogo, se da como presupuesto de que trasmitir la fe equivale a enseñar la doctrina cristiana, con el fin de iniciar a los alumnos en la "plenitud de la vida cristiana" (Catecismo, 1992; p. 14). Entonces "Catequesis", según los postulados de la Iglesia Católica Romana, significa el conjunto de esfuerzos por parte de la Iglesia para formar discípulos; lo cual se logra por medio de la transmisión y educación de la doctrina católica. Más allá de la incursión de los niños y jóvenes en la doctrina, el catecismo expone, como uno de los objetivos más importantes, la formación de una conciencia moral, pues, sólo mediante la rectitud de tal conciencia se puede alcanzar la dignidad de la persona bumana. Ella tiene como función establecer el juicio moral. De acuerdo con el Catecismo, la formación de la conciencia moral se puede resumir de la siguiente manera:

"La palabra de Dios es una luz para nuestros pasos, es preciso que la asimilemos en la fe y la oración, y la pongamos en práctica. Así se forma la conciencia moral" (Catecismo; 1992, p. 408).

En síntesis: la posición de la Iglesia Católica, respecto a la educación religiosa, es de que ésta consiste en la transmisión de su doctrina, la cual, además, conlleva una función formadora de conciencia moral.

La postura de un estado católico, apostólico y romano no puede ni debe ser diferente de las directrices planteadas por la institucionalidad eclesiástica ${ }^{2}$. La situación se plantea de la siguiente manera: La institucionalidad católica concibe que la educación de la fe se iguala a la transmisión de la doctrina de la Iglesia Católica; y que esta transmisión permite, entonces, entre otros aspectos, la formación de la conciencia moral. Esta permite, a su vez, formular juicios morales, acertados en la medida en que correspondan al conocimiento de los dogmas religiosos católicos.

De esta formulación se desprenden al menos dos objetivos, denominados por la Iglesia Católica "enseñanza de la fe":

- El conocimiento de su doctrina.

- $\quad$ Y, la formación de la moral.

Es pertinente hacer ver cómo los propósitos de la educación religiosa de Costa Rica no están muy lejanos de los lineamientos propios de la Iglesia Católica; elementos que aparecen centrales en los diferentes ciclos de los programas de educación religiosa (Ministerio de Educación Pública; 1996).

La presencia del elemento moralizador, en pro de una educación religiosa, tanto formal como informal, no es de extrañar, si se conside, de acuerdo con Küng (1986), que la moral, en conjunto con la doctrina y los ritos, constituyen las funciones fundamentales de la religión. Estas funciones son además, siguiendo al mismo autor, el lugar en donde se establece la relación con lo trascendente. Justamente, en 
estos términos es definida la religión. Por ello, una educación religiosa centrada en transmitir la doctrina y en presentar un afán moralizador; ambos coherentes como función propia y constitutiva de cualquier religión.

Todo lo anterior proporciona un marco para plantear el problema, razón de ser de este ensayo: ¿Cómo presentar una educación religiosa desde una postura científica, en donde se puedan abarcar los dos puntos que más resaltan en las propuestas educativas ya comentadas: la formación en la doctrina y la fe, y la formación moral?

La segunda parte de la pregunta no dista mucho de lo que planteaba, en la Sorbona, Durkheim (1922) a principios de siglo. En este texto, se hace el análisis de cómo separar la educación moral de la Iglesia mediante una racionalización de la moral. Esto ya fue respondido con solvencia mediante las propuestas de Lawrence Kohlberg al aplicar su modelo de desarrollo moral a la práctica educativa.

Sus ideas son tomadas como complemento necesario para la línea de trabajo, en la esfera de educación religiosa, sobre la cual planteo algunas reflexiones desde la psicología de la religión.

Para responder a la pregunta, se toman en cuenta los planteamientos de la psicología de la religión, a partir de dos supuestos que surgieron desde lo revisado en párrafos anteriores sobre la educación religiosa:

1. Una educación religiosa, basada sobre la transmisión, a los individuos, de doctrinas como punto de partida para el fomento de la fe, está destinada, en gran medida, a fracasar, pues, el desarrollo de la fe no está ligado, única y exclusivamente, al conocimiento de la doctrina religiosa por parte de tales individuos. Esto también tiene que ver con la permutación de la vivencia de lo supuesto trascendente en el curso del desarrollo humano en sus diferentes niveles.

2. Una educación religiosa no es garante de una educación moral o de una formación de la conciencia moral, por lo que, en este segundo aspecto, la educación religiosa terminará por ser inadecuada.

\section{Aspectos centrales de la psicología de la religión}

La psicología de la religión consiste en una rama de la psicología dedicada al estudio de la incidencia del fenómeno religioso en la vivencia de las personas, y la experiencia de tal fenómeno por parte de éstas mismas. Por eso, la psicología de la religión estudia las creencias, los sentimientos, y, en general, la relación del sujeto con el fenómeno religioso. Esta concepción es tomada de autores, entre otros, Vergote (1993), quien propone que la psicología de la religión debe abocarse al estudio de lo estrictamente psicológico en el fenómeno religioso.

Con tal postura, está haciendo referencia al sujeto y a su relación con lo trascendente, y considera, a la vez, el contexto en el que éste se encuentra. De esta manera, se establecen límites al objeto de estudio con respecto a otras áreas de la ciencia que podrían abocarse al estudio de este mismo fenómeno.

En términos epistemológicos, el estudio de la psicología de la religión se centra en lo que Jaspard (1995) denominó O”. Dentro de tal esquema (ver esquema 1), para comprender la fe religiosa, O" significa la representación interna del objeto de la trascendencia (O), en esta mediación -de carácter simbóliCO-, O' es la representación cultural: la "transposición metafórica de lo divino que le otorga un aspecto antropomórfico" (Jaspard;1995, p. 5). Las diferentes representaciones del objeto están, a su vez, articuladas mediante dos tipos de creencia, en las cuales, la creencia 2 (Cr2), válida o no, de forma subjetiva la mediación O', mientras la creencia 1 (Cr1) representa el reconocimiento de que $\mathrm{O}$ existe, y la creencia de que O' está referida a O.

De esta manera, la psicología de la religión se deberá de preocupar -como ya se mencionó- de la O”, así como también de las 
creencias que se desprendan en las mediaciones del supuesto objeto trascendente.

\section{Esquema 1}

Mediaciones simbólicas y creencias según Jaspard

$$
\mathrm{S}-\mathrm{O} \text { " - O' }-\mathrm{O}
$$

Este panorama general de la psicología de la religión, específicamente, de la psicología del desarrollo religioso, plantea, desde ya, una diferente visión en el estudio de lo religioso desde la psicología.

El interés, entonces, no va a radicar en los contenidos o manifestaciones culturales, sino en la forma como lo religioso sea vivido por los sujetos, independientemente de si esta representación interna del objeto (O") es revestida por el investigador con características más cognitivas o emocionales.

De acuerdo con Gorsuch (1988), las aproximaciones teóricas a la comprensión del desarrollo religioso se han orientado desde tres abordajes: Las teorías de la proyección, las teorías de la socialización y las teorías del desarrollo cognoscitivo.

La primera aproximación teórica ha investigado el fenómeno, principalmente, mediante las relaciones entre el concepto de Dios y las figuras parentales (Vergonte \& Tamayo; 1980). Por otro lado, las teorías de la socialización investigan el impacto de los otros en el individuo durante el desarrollo.

El principal problema de las teorías planteadas, desde esta aproximación teórica, es de que, aunque se haya encontrado una correlación entre la escogencia religiosa de los hijos y la religión de los padres, la escogencia de la escuela y la asistencia a la enseñanza parroquial, entre otras, no se puede hablar de una relación causal entre estas variables, por lo que, desde esta perspectiva, los resultados de las investigaciones carecen de la solidez esperada.

Las teorías de desarrollo cognitivo se han planteado el problema de la búsqueda del entendimiento de las formas de ver el mundo por las personas, en el desarrollo acerca de aspectos como la oración, Dios, etc.
Los resultados se han orientado, sobre todo, a confirmar los planteamientos piagetanos; y el principal problema, la ausencia de integración en los resultados de investigación.

Reich (1993) planteó la necesidad de efectuar una integración de las diferentes teorías del desarrollo religioso, por cuanto la pluralidad de discursos, desde las diferentes posiciones teóricas, genera una incomunicabilidad, la cual repercutirá en la imposibilidad de generar propuestas de índole general.

El planteamiento de una teoría integrada del desarrollo religioso debe contener, según Reich (1993), cinco especificaciones básicas:

1. Que se refiera a un proceso psíquico que ocurre en el organismo.

2. Que el desarrollo sea caracterizado por una gradual coordinación de los individuos con lo biofísico, lo sociocultural y la realidad espiritual, de manera que se pueda explicar la relación entre las fuerzas internas y externas en el curso del desarrollo.

3. Que tome en cuenta los contextos sociales, en donde el desarrollo ocurre, y las maneras como estos contextos sean relatados en las actitudes, las conductas, el desarrollo.

4. Que tome en cuenta las características universales del desarrollo, como de las diferencias individuales.

5. Que especifique los mecanismos mediante los cuales el cambio ocurre en el desarrollo, y explique los factores que inciden en favor de la alta religiosidad, incluidos la conversión y la apostasía.

Diferentes investigaciones, en la esfera del desarrollo religioso (específicamente, dentro del desarrollo cognoscitivo) han arrojado datos en relación con la primera premisa (1.), de la cual parte este escrito. Se ha encontrado que es hasta la adolescencia cuando se puede afirmar que las personas están en capacidad de comprender las expresiones religiosas abstractas (Elkind, 1981; Oser \& Gmünder; 1998). 
Con estos datos se está en grado de afirmar, entonces, que el aprendizaje de las representaciones religiosas, anterior a la adolescencia, va a tener características de un aprendizaje acumulativo de información. Aunque se trate de hacer este aprendizaje significativo a favor del individuo (introduciendo prácticas o discusiones sobre la cotidianidad y la aplicación de los preceptos religiosos a estas áreas), el niño no posee las condiciones cognoscitivas necesarias para una comprensión real de lo religioso.

Ante condiciones deductivas, uno de los problemas que se puede presentar es que el sujeto del desarrollo equipare las diferentes mediaciones simbólicas de lo trascendente (O”, O') con lo trascendente mismo (O). Tal problema ya fue señalado por Jaspard (1995). De acuerdo con éste, se puede tener el riesgo de asumir una posición antropomórfica de la mediación de lo trascendente, en lugar de adoptar una posición simbólica, allí en donde se daría, en forma normal, la mediación.

La formación religiosa orientada a que el sujeto conozca un sistema de creencias, puede generar sujetos bien informados respecto a la doctrina, pero, probablemente, con escasas posibilidades de elaborar una autocrítica o una crítica hacia sus creencias.

Es probable que el desarrollarse dentro de un ambiente que le propone al sujeto las respuestas correctas, sobre la manera de interpretar un fenómeno religioso, termine por atrofiar, en no pocos casos, las posibilidades de un desarrollo autónomo de la mediación subjetiva (O"). Sin embargo, en la adolescencia, se pueden esperar cuestionamientos a los contenidos recibidos, durante los diferentes momentos de la instrucción en la fe religiosa. Esto remitiría, nuevamente, a la vivencia de lo trascendente de una manera no paralela a la permeabilidad del texto de la Iglesia. Ciertamente, esta es una interpretación posible dentro de otras.

Hutsebaut \& Verhoeven (1991), en estudios longitudinales con adolescentes, señalaron que la edad de los quince años resulta ser crucial en cuanto a la práctica religiosa y la creencia en la existencia de Dios se refiere.
En sus datos, se constata un decrecimiento en la creencia al mismo tiempo que en la práctica religiosa; por otra parte, se observa en aumento a otros que se presuponen dudosos en cuanto a la existencia de Dios o son ateos.

En nuestro contexto, estos datos se interpretan como un problema de la sociedad actual; sin embargo, se deja de lado la posibilidad de asumir que éste es el resultado natural de una educación religiosa centrada en el uso de estrategias persuasivas sobre los sujetos, más que propiciar el desarrollo natural de estos respecto del fenómeno religioso. Por esto, no se puede hablar de un desarrollo autónomo de la vivencia religiosa.

Sintetizando, señalo que el aprendizaje de los contenidos doctrinales de una religión no significa, necesariamente, que éste tenga una modificación sustancial en la vivencia de la fe.

\section{Bases de la educación moral y crítica a la educación tradicional}

La teoría del desarrollo moral, propuesta por Lawrence Kohlberg, no sólo ha influido sobre la psicología y la filosofía; también, de manera muy importante, ha tenido una gran relevancia en el ámbito educativo, área en donde propuso aplicar su teoría del desarrollo moral. El mismo Kohlberg ha planteado:

"En esta concepción de educación moral, la meta es la estimulación del desarrollo "natural" del juicio moral de cada uno de los niños y de las capacidades para que ellos por sí mismos sean capaces de controlar sus conductas" (Kohlberg; 1981, 300)3.

Así, el objetivo de la educación se puede ver como el desarrollo natural de lo que de por sí existe en los individuos.

En sus investigaciones, Kohlberg encontró que, de forma universal, el individuo atraviesa por seis estadios de desarrollo en el razonamiento moral ${ }^{4}$.

Por medio de las investigaciones transculturales realizadas por él mismo y colaboradores, no se encontró evidencia de que 
los niños de diversas culturas y de diferentes religiones manifestaran razonamientos morales distintos. Por el contrario, se constató que, independientemente del sistema de creencias religiosas, los niños atravesaban por los mismos estadios de juicio moral, por lo que, para Kohlberg, se sigue, de forma universal, la misma estructura. Si bien el ritmo de avances en la secuencia de estadios puede variar de acuerdo con las posibilidades que la relación individuo-cultura presente, la secuencia en los estadios es la misma.

En lo referente a la educación moral: a la aplicación de los resultados de investigación en el ámbito educativo, se planteó y probó que, si se estimulaba a un grupo de estudiantes -a quienes previamente se había evaluado en su desarrollo moral-, para conducirlos a razonamientos en estadios inmediatamente superiores a su nivel de razonamiento moral, entonces ellos, luego de algunas sesiones de trabajo, podrían alcanzar, en modo permanente, esta forma de razonamiento. Dichos hallazgos fueron denominados "Efecto Blatt", en nombre del estudiante que expuso tal posibilidad.

Tales investigaciones demostraron lo siguiente:

1. Que el desarrollo moral responde a la intervención educativa, de manera que el avance de estadios, que generalmente conlleva un lapso extendido, puede darse en períodos más concentrados.

2. Que el efecto del método aplicado no fue temporal, mas bien permanente, por lo cual no se dio ningún aprendizaje de "respuestas correctas" sino del razonamiento de estadios inmediatamente superiores al que el estudiante se encontraba. Esto fue confirmado mediante evaluaciones a los grupos de estudiantes en momentos posteriores a los espacios de intervención pedagógico-morales.

3. Que el desarrollo estimulado se produce, cuando la intervención establece las condiciones que promuevan el paso a otra etapa, entre ellas: proporcio- nar oportunidades para el conflicto cognitivo, el desarrollo de la conciencia moral, la asunción de papeles y el acceso a una forma de razonamiento moral que se encuentre por encima de la propia etapa (Kohlberg, et al.; 1997).

Ante estos hallazgos, Kohlberg inicia sus estudios sobre educación moral, aunque encuentra que las posibilidades de desarrollar una educación moral se podían dificultar debido al "curriculum oculto5".

Para introducir el desarrollo moral en las escuelas, una de las formas propuestas por él, es el establecimiento de escuelas democráticas.

Otras formas de aplicar la teoría del desarrollo moral a la escuela, son las expuestas por Edelstein (1989).

Este autor plantea cuatro tipos posibles de aplicación de la pedagogía moral:

El primero consiste en discutir, ocasionalmente, dilemas socio-morales de acuerdo con los hechos o circunstancias que tengan lugar en la clase y al exterior de ésta.

El segundo plantea que la discusión sea impartida en, forma integrada, en todas las diferentes materias con miras a realizar, constantemente, intervenciones de tipo pedagógico moral.

El tercero propone crear cursos, tanto dentro como fuera de la escuela, a fin de estimular el juicio moral, así como incorporar temas relacionados con valores.

El cuarto corresponde a lo que Kohlberg llamó la comunidad justa (Kohlberg, et al, 1997).

Los aportes anteriores, propuestos para mejorar la educación moral, no han sido bien recibidos en el ámbito religioso. Claro ejemplo son los señalamientos de los sectores religiosos de Los Estados Unidos, quienes expresaban que la educación moral estaba pretendiendo ocupar el lugar que le correspondía a la educación religiosa. Esto generó que se dieran diferentes investigaciones, en donde se relacionan el desarrollo moral y la religión; en especial, el papel de la educación religiosa en el desarrollo moral. 
Ante tal panorama, la relación hallada por Kohlberg es de que la influencia de la educación religiosa es muy limitada, aunque aclara:

“...no estoy diciendo que la educación religiosa es incapaz de jugar algún rol en el desarrollo moral. Argumento, que la educación religiosa formal no es específicamente importante o la única que juega un rol en el desarrollo moral." (Kohlberg, 1981, p. 304) ${ }^{6}$.

Que la educación religiosa no sea especialmente significativa en el desarrollo moral -no propicia un desarrollo moral diferente a lo que podría hacer cualquier otro tipo de lección en las escuelas- en Los Estados Unidos, no significa, sin embargo, que con otros modelos educativos no se alcancen avances significativos.

En el ámbito técnico, sin embargo, no se espera que el avance, en el desarrollo moral, se logre con modelos educativos que centren su atención en una perspectiva de acumulación de conocimientos y aprendizaje de normas, en las cuales podría ser predominante el elemento el culpógeno.

Se puede resumir el principal aporte de Kohlberg, en pro de la educación, en la posibilidad de una educación moral basada sobre la teoría psicológica del desarrollo moral. A esto se puede agregar que esta educación moral esté orientada científicamente, y pueda ser independiente de las diferencias sociales, étnicas, religiosas y, en general, culturales.

Sobre la base de los resultados de las investigaciones en desarrollo moral, la educación religiosa no hace ningún aporte significativo a favor del desarrollo del sujeto, por lo que, si se desean lograr efectos, en este ámbito del desarrollo humano, lo adecuado es prescindir de la educación religiosa tradicional como panacea de los males morales del individuo y de la sociedad.

El empleo de estrategias de intervención, en esta área, como los modelos psicológicos de la moral, plantean la posibilidad de formar un individuo capaz de reflexionar en torno a los diferentes dilemas morales que se tope en su vida cotidiana, sin necesidad de evaluarlos desde aspectos normativos, los cuales, además, se fundamenten en la "culpabilización" o las amenazas a la posibilidad de la pérdida de la vida con lo trascendente ${ }^{7}$.

El fomento de una educación moral, fundamentada en la racionalidad y no en el seguimiento de una determinada doctrina religiosa o de una ley divina --por lo tanto incuestionable-, podría dar paso al desarrollo de "yoes" autónomos, que sean, a su vez, sociales.

Esta posición podría parecer, en algún sentido, paradójica, mas no lo es, si se piensa que esto se basa sobre la propuesta de Piaget (citado por Kohlberg, et al.: 1997), en done el yo autónomo es creado y sostenido por otros "yoes" autónomos. En este sentido, se alude a la autonomía basada sobre la cooperación, y no en el individualismo.

\section{Aspectos mínimos para desarrollar una nueva educación religiosa}

Es importante señalar que los siguientes puntos no necesariamente van a ser los únicos; mas sí son, a mi parecer, indispensables para que se configure una educación religiosa con las características que he venido delineando.

Las premisas básicas, para establecer una educación religiosa orientadas por la psicología, son cinco:

1. Deben ser formuladas desde la premisa de que el desarrollo humano religioso es independiente de los contenidos doctrinales de los diferentes grupos religiosos.

2. La educación religiosa debe ser presentada desde la base de una búsqueda de universalidad, de manera que sea capaz de llegar a toda la población y no sólo a la seguidora de la religión oficial del país.

3. Una educación religiosa, desde la psicología de la religión, debe basarse sobre el desarrollo de la vivencia personal con respecto al fenómeno de lo religioso, más que en la acumulación de conocimientos de la doctrina de una determinada religión. 
4. Se debe fundamentar sobre una perspectiva del desarrollo humano religioso, el cual presente una visión integrada de éste: contemplar tanto la evolución cognoscitiva de los sujetos como el desarrollo humano de los afectos respecto a lo religioso.

5. Dentro de los aspectos de contenido, es importante no ofrecerle a la persona una visión de conocimiento acabado: una doctrina dogmática, sino, más bien, abrir las oportunidades, para que el individuo pueda desenvolver una perspectiva amplia e integrada de la esencia de las diferentes religiones; visión por ser presentada desde una visión religiosamente neutra; de manera que el sujeto pueda formar su propio criterio respecto a las doctrinas.

Sobre el quinto punto, he aquí algunos aspectos básicos dignos de tomar en cuenta dentro de una propuesta curricular:

- Incluir el conocimiento de diferentes posiciones respecto a lo religioso, el estudio de las diferentes religiones y no sólo de la religión mayoritaria del país. A tal fin, explorar, en diferentes aspectos y posiciones: historia de las religiones, sus elementos políticos y los fundamentos doctrinales. Eso no se vislumbra para lograr un desarrollo de la $\mathrm{fe}$, sino para ofrecer un marco general sobre las posibilidades de entender la experiencia religiosa.

- Contemplar el estudio de las religiones desde la perspectiva de su influencia en las diferentes áreas de la vida social: el arte, la política, la economía, lo cotidiano, etc.

- Dentro del estudio de las diferentes religiones, enfatizar el análisis de las distintas visiones religiosas del mundo de grupos autóctonos del continente americano, prioritariamente, las de los grupos propios del país.
En lo referente a la moral, la otra gran área planteada como quasi constitutiva de la educación religiosa, la educación, desde una perspectiva moral, es considerada como inevitable dentro de la educación religiosa en sus diferentes manifestaciones tanto formal como informal.

Si bien es cierto que existen diferentes aproximaciones, para generar el desarrollo moral, la educación moral puede desligarse, por completo, de la educación religiosa, especialmente, si se toman en cuenta los elementos en párrafos arriba señalados.

Una opción atractiva es poner en marcha, en las instituciones educativas, los modelos de comunidad justa; en este sentido, de que se realicen discusiones en pro del desarrollo moral de los educandos, independientemente de un espacio específico. Eso constituye la opción más adecuada, la cual permite, además, ejecutar el razonamiento moral sobre diferentes dilemas tanto cotidianos como sobre los de la materia que se estudie pueda proporcionar. En tal caso, las clases de educación religiosa conformarán un espacio más, para que esto se ejecute.

Con todo, igualmente, las clases de educación religiosa pueden convertirse, también en el espacio ideal para desarrollar la educación moral en los estudiantes; esencialmente, por la relación histórica existente entre moral y religión. De esta manera, el programa de estudios de educación religiosa puede ser concebido como una integración de ambos aspectos: desarrollo religioso y desarrollo moral; con la diferencia de que ambos estarían científicamente orientados.

\section{Consideraciones finales}

La propuesta anterior bien puede ser claramente calificada en el ámbito de lo utópico, y no porque sea inoperante en sí misma, sino, porque es imposible obviar la realidad de un medio social y político, en donde resulta difícil concretar una educación religiosa con tales características. 
La educación religiosa costarricense posee bases histórico-políticas que se han convertido en la materialización de derechos legales insalvables. La integración de tal tipo de educación, data, en Costa Rica, de 1940 mediante el concurso del Dr. Rafael Ángel Calderón Guardia, quien, el 8 de diciembre, emitió la Ley \#21, donde establece la educación religiosa como parte de la educación pública.

Pero más allá de estos aspectos, una educación religiosa alejada y ajena de los lineamientos de la oficialidad religiosa, encontrará un sin número de barreras y trabas casi infranqueables; pues, en un país, en donde la institución religiosa posee la potestad de decidir sobre la forma y contenido de los textos de educación ${ }^{8}$, y, sobre todo, de contenidos educativos que no se relacionen con lo estrictamente religioso, ¿qué no se podría objetar contra una educación religiosa, donde el papel desempeñado por la Iglesia ya no sea más el principal?

Existen otras condiciones no menos desalentadoras: la formación docente, en Costa Rica, no siempre es lo suficientemente sólida como podría esperarse; lo cual no es de extrañar, si se considera que el sistema educativo costarricense vive un proceso de resquebrajamiento que lo ha inducido a una carencia de evolución; aspecto, éste, que, necesariamente, se relaciona con el desempeño y la calidad de la formación de los docentes en los diferentes ciclos educativos, como son: malas condiciones laborales, sueldos bajos, pérdida del prestigio de la profesión, una formación docente de corto plazo y un largo número de circunstancias constituyen la causa de que, aún con el mejor programa educativo en cualquier área académica, se deben tomar en cuenta las condiciones que confabularán para dificultar su ejecución.

Un docente apto para desarrollar, en sus estudiantes, un programa constituido a partir de los elementos de mi propuesta, necesariamente ha de conocer de teosofía, sociología de la religión, historia de las religiones, psicología de la religión, psicología del desarrollo humano, además de pedagogía, didáctica, etc.

Aunque no se requiera ser un experto en ninguna de estas áreas, en el contexto educativo pensar en un manejo integrado y aceptable, necesariamente remite a una carrera universitaria más extensa que la actual.

Pero lo anteriormente señalado no implica, de ninguna manera, que se deba rechazar ad portas la posibilidad de mejorar el sistema educativo costarricense en la disciplina de religión. Pero, debe alertar que modificaciones, como las propuestas, constituyen la necesidad de estar dispuestos a modificar las bases sobre las que se ha construido, y descansa aún, en Costa Rica, el proyecto educativo.

Dentro de los elementos, desde los cuales se puede establecer una educación religiosa, lo precedentemente bosquejado por mí conducirá a impartir una educación con características universalistas, independientemente del grupo religioso al que esté adherido el estudiante, de su opción respecto a la creencia o la ausencia de ésta, de la cultura o subcultura a la cual pertenezca y de su grupo etáreo; todo lo cual será también, paradójicamente, una educación religiosa sin una doctrina religiosa exclusiva de adscripción a tal o cual religión.

El que sea impartida, en la instrucción pública, una educación religiosa con una orientación, única y exclusivamente católica, es producto de un estado que pretende y desea proyectarse con tal visión y opción religiosas.

Finalmente, cabe plantearse, aquí, los siguientes cuestionamientos: El estado, al optar por determinada doctrina religiosa, ¿acaso no está haciendo, en forma inmediata, un acto de discriminación contra quienes no sigan tal o cual doctrina, aún cuando admita la existencia de estos?; ¿la educación religiosa, orientada desde una única óptica puramente doctrinal, ¿no constituye, acaso, la escenificación de tal supuesto acto discriminatorio?

No obstante, es absurdo plantear una única respuesta a tales preguntas, lo cual no excluye, de ninguna manera la existencia de otras posiciones y opciones. En lo tocante a los fines propuestos de este ensayo: una respuesta positiva a estas dos formulaciones, la presente propuesta constituye, de por sí, una tentativa de solución al problema planteado. 


\section{Notas}

1. De acuerdo con el Estado de la Nación (1995), en los programas de rescate de valores, se trata de exhaltar los principios religiosos y cristianos, así como los derechos humanos y la solidaridad.

2. El Catecismo de la Iglesia Católica trata una enorme cantidad de temas; sin embargo, es de interés de los fines de este ensayo el centrarse en estos dos puntos.

3. Traducción libre del autor.

4. Existen críticas a la propuesta de universalidad de la teoría del desarrollo moral. Una buena exposición al respecto se puede encontrar en Stinger y Schweder (1990). Una reflexión sobre el tema de la universalidad en población Bribri costarricense se encuentra en Rodríguez (2000).

5. Como currículum oculto, Kohlberg designa la diferencia dada entre los razonamientos que se puedan desarrollar como producto del efecto Blatt, y las normas y conductas en la cotidianidad de la escuela; la no correspondencia podría afectar el desarrollo moral. Sus preocupaciones llevaron a proponer, entre otras cosas, la necesidad de la evaluación de la cultura moral de las instituciones educativas.

6. Traducción libre del autor

7. Me refiero en particular al cristianismo en relación con el pecado y aspectos como la confesión, y las condiciones de la salvación.

8. Estoy pensando aquí en la elaboración de las guías de educación sexual.

\section{Referencias bibliográficas}

Catecismo de la Iglesia católica. Santo Domingo: Librería Juan Pablo II, 1992.

Constitución Política de la República de Costa Rica. San José: Imprenta Nacional, 1993.

Durkheim, E . La educación moral. Buenos Aires: Losada, 1922.

Edelstein, W. "Fomento del desarrollo moral en la escuela. Alcances y límites". Educación. No 40, 1989.
Elkind, D. El niño y la realidad. Tres aspectos del desarrollo. Paidos: Barcelona, 1981.

Gorsuch, R. "Psychology of religion". Annual Reviews in Psychology. № 39, 1988.

Hutsebaut, D; Verhoven, D. "The adolecents representation of god from age 12 to 18". Journal of empirical theology. 우, Vol. 1, 1991.

Jaspard, J. “¿Qu'est-ce que croire? Repères épistémologiques pour situer croyance et foi dans le contexte religieux". Catéchèse. París, n¹39, Vol. 2, 1995. ¿Qué es creer? Lineamientos epistemológicos para situar creencia y fe dentro del contexto religioso. Trad. Quirós, A.

Kohlberg, L. "Moral and religious education and the public schools: A developmental view". En: Kohlberg, L. (1981) The Philosophy of moral development: Moral stages and the idea of justice. San Francisco: Harper \& Row. 1981.

Kohlberg, L; et al. La educación moral. Barcelona: Gedisa, 1997.

Ministerio de Educación Pública. Programa de Estudios de Educación Religiosa. Ciclos I, II, III, y Educación Diversificada. San José Costa Rica, 1996.

Oser, F.; Gmünder, P. El hombre. Estadios de su desarrollo religioso. Barcelona: Ariel, 1998.

Proyecto estado de la nación. San José, Costa Rica, 1995.

Reich, H. "Integrating differing theories. The case of religiosus development". Journal of Empirical Theology. n6, Vol.1, 1993.

Rodríguez, J.M. Gramática de la moral. La adjetivación de las acciones en los Bribris. Tesis para optar por el grado de licenciatura en psicología. Escuela de Psicología, Universidad de Costa Rica, 2000. 
Stiger, J. Schweder, R. Cultural Psychology: Enssays on comparative human development. Massachusetts: Cambridge University Press, 1990.

Vergote, A. "What the psychology of religion Is and what it is not". The Jour- nal For The Psychology of Religion. N³, Vol.2, 1993.

Vergote, A.; Tamayo, A. The parental figures and the representation of God. Bélgica: Leuven University Press, 1980. 